\title{
Two Statistics Linking Dyck Paths and Non-crossing Partitions
}

\author{
Haijian Zhao and Zheyuan Zhong \\ Center for Combinatorics, LPMC-TJKLC \\ Nankai University, Tianjin, P.R. China \\ haijian.nankai@gmail.com, zhongzheyuan@gmail.com
}

Submitted: Dec 6, 2010; Accepted: Apr 1, 2011; Published: Apr 7, 2011

Mathematics Subject Classifications: 05A15, 05A18

\begin{abstract}
We introduce a pair of statistics, maj and sh, on Dyck paths and show that they are equidistributed. Then we prove that this maj is equivalent to the statistics $l s$ and $r b$ on non-crossing partitions. Based on non-crossing partitions, we give the most obvious $q$-analogue of the Narayana numbers and the Catalan numbers.
\end{abstract}

\section{Introduction and Notation}

A Dyck path of length $2 n$ is a path in $\mathbb{N} \times \mathbb{N}$ from $(0,0)$ to $(2 n, 0)$ using steps $U=(1,1)$ and $D=(1,-1)$, which never goes below the $x$-axis. The $U$ steps and $D$ steps are called up steps and down steps respectively. The set of all Dyck paths of length $2 n$ is denoted by $\mathscr{D}_{n}$. It is well known that the cardinality of the set $\mathscr{D}_{n}$ is the $n$-th Catalan number

$$
C_{n}=\frac{1}{n+1}\left(\begin{array}{c}
2 n \\
n
\end{array}\right) .
$$

It will be convenient to code a Dyck path $w$ in the letters $\left\{U_{i}\right\}_{i=1}^{\infty} \cup\left\{D_{i}\right\}_{i=1}^{\infty}$ by letting $U_{i}$ and $D_{j}$ stand for the $i$-th up step and the $j$-th down step in $w$, respectively.

If $D_{i} U_{j}$ (resp., $U_{i} D_{j}$ ) are two successive letters in $w$, then we call the subword $D_{i} U_{j}$ (resp., $\left.U_{i} D_{j}\right)$ a valley (resp., peak). Meanwhile if $U_{i-1} U_{i} D_{j}$ (or $D_{i-1} D_{i} U_{j}$ ) are three successive letters in $w$, then we call $U_{i-1} U_{i} D_{j}$ (or $D_{i-1} D_{i} U_{j}$ ) a skew hook.

The set of Dyck paths of length $2 n$ with $k$ valleys will be denoted by $\mathscr{D}_{n, k}$. It is well known that the cardinality of $\mathscr{D}_{n, k}$ is given by the Narayana number

$$
\frac{1}{n}\left(\begin{array}{c}
n \\
k+1
\end{array}\right)\left(\begin{array}{l}
n \\
k
\end{array}\right) .
$$


Definition 1.1 Let $w$ be any Dyck path of length $2 n$, then the descent set of $w$ is

$$
\mathrm{D}(w):=\left\{i \mid D_{i} U_{j} \text { forms a valley in } w\right\} .
$$

For a given Dyck path $w$, we define the statistic maj $(w)$ by

$$
\operatorname{maj}(w):=\sum_{i \in \mathrm{D}(w)} i .
$$

The maj defined on Dyck paths here is different from that defined in [4]. To distinguish these two majors, we use Maj to denote the one defined in [4].

Definition 1.2 Let $w$ be any Dyck path of length $2 n$, then the skew hook set of $w$ is

$\mathrm{H}(w):=\left\{j \mid U_{i-1} U_{i} D_{j}\right.$ is a skew hook of $\left.w\right\} \cup\left\{i \mid D_{i-1} D_{i} U_{j}\right.$ is a skew hook of $\left.w\right\}$.

For a given Dyck path $w$, we define the statistic $\operatorname{sh}(w)$ by

$$
\operatorname{sh}(w):=\sum_{i \in \mathrm{H}(w)} i
$$

Example 1.3 Let $w$ be the Dyck path in Figure 1, then $\operatorname{maj}(w)=2+4=6$ and $\operatorname{sh}(w)=1+2+3+4=10$.
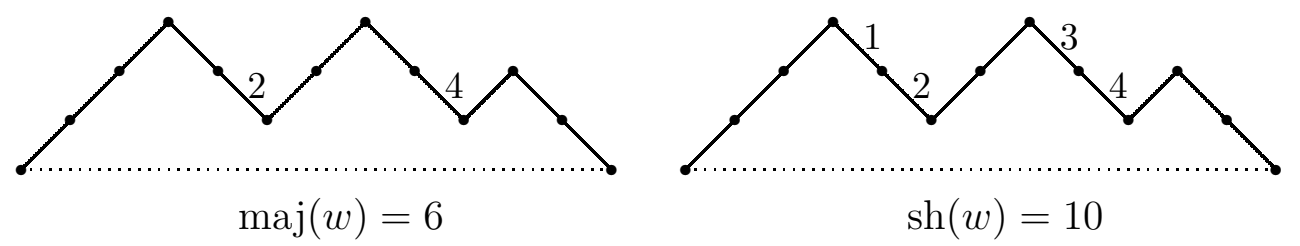

Figure 1: $\operatorname{maj}(w)$ and $\operatorname{sh}(w)$.

A partition of $[n]:=\{1,2, \ldots, n\}$ is a collection of disjoint nonempty subsets of $[n]$, called blocks, whose union is $[n]$. We denote by $\Pi_{n}$ the set of all partitions of $[n]=$ $\{1,2, \ldots, n\}$. A standard way of writing a partition $\pi$ with $k$ blocks is $\pi=B_{1} / B_{2} / \cdots / B_{k}$, where the blocks are ordered in the increasing order of their minimum elements and within each block, the elements are written in the numerical order. When appropriate, we will emphasize that $B_{i}$ is a block of the partition $\pi$ by writing $B_{i}(\pi)$.

A partition $\pi=B_{1} / B_{2} / \cdots / B_{k}$ of $[n]$ is non-crossing if whenever a quadruple of elements $1 \leq a<b<c<d \leq n$ satisfies $a, c \in B_{i}$ and $b, d \in B_{j}$ for some $1 \leq i, j \leq k$, then in fact $i=j$; thus, the blocks do not "cross".

The set of all non-crossing partitions of $[n]$ will be denoted by $N C(n)$, and the set of non-crossing partitions of $[n]$ into $k$ blocks will be denoted by $N C(n, k)$.

A partition $\pi \in \Pi_{n}$ may be represented via its restricted growth function [5] ("RG function"), $w:[n] \rightarrow[n], w(i)=$ the index of the block of $\pi$ which contains $i$. In this 
paper the restricted growth function associated with $\pi \in \Pi_{n}$ will be the word $w=$ $w_{1} w_{2} \cdots w_{n}$, where $w_{i}=w(i)$.

Given a partition $\pi=B_{1} / B_{2} / \cdots / B_{k}$ of $[n]$, let $w=w_{1} w_{2} \cdots w_{n}$ be its restricted growth function. Simion [7] defined two statistics on partitions:

$$
l s(\pi):=\sum_{i=1}^{n} \#\left\{w_{j} \mid w_{j}<w_{i}, j<i\right\},
$$

and

$$
r b(\pi):=\sum_{i=1}^{n} \#\left\{w_{j} \mid w_{j}>w_{i}, j>i\right\} .
$$

For example, if $n=9$ and $\pi=157 / 26 / 34 / 89$, then $w=123312144$, and $l s(\pi)=0+1+2+2+0+1+0+3+3=12$, and $r b(\pi)=3+2+1+1+2+1+1+0+0=11$.

Here we list some known results about the statistics $l s$ and $r b$ which are very useful in our later proof. For the details, please see [7].

Lemma 1.4 Let $\pi=B_{1} / B_{2} / \cdots / B_{k}$ be any partition of $[n]$. Then

$$
l s(\pi)=\sum_{i=1}^{k}(i-1)\left|B_{i}\right| .
$$

Lemma 1.5 Let $\pi=B_{1} / B_{2} / \cdots / B_{k}$ be a non-crossing partition of $[n]$. Then

$$
r b(\pi)=\sum_{i=1}^{k}\left(m_{i}(\pi)-1\right),
$$

where $m_{i}(\pi)=\min \left\{a: a \in B_{i}\right\}$.

The $q$-analogue for integer $n \geq 1$ is $[n]=1+q+\cdots+q^{n-1}$, and $[n] !=[n][n-1] \cdots[1]$ for $n \geq 1$ with $[0] !=1$. The $q$-binomial coefficient is

$$
\left[\begin{array}{l}
n \\
k
\end{array}\right]=\frac{[n] !}{[k] ![n-k] !} .
$$

The rest of this paper is organized as follows. In Section 2, we prove that the statistics maj and sh are equally distributed on Dyck paths. In Section 3, we construct two bijections between Dyck paths and non-crossing partitions which respectively send the major index on Dyck paths to the $l s$ index and $r b$ index of the corresponding non-crossing partitions. The objective of Section 4 is to give the most obvious $q$-analogue of the Narayana numbers and the Catalan numbers using the statistics $l s$ and $r b$ on non-crossing partitions. 


\section{Equidistribution of maj and sh}

First of all, we point out that the two statistics maj and sh have the same distribution over $\mathscr{D}_{n}$, which can be derived from the bijection given by Benchekroun and Moszkowski [2]. For a better understanding of the equidistribution of maj and sh, we give an iterative construction of the bijection, which is constructed recursively by Benchekroun and Moszkowski.

Theorem 2.1 There is a bijection $\rho: \mathscr{D}_{n} \rightarrow \mathscr{D}_{n}$ such that maj $(w)=\operatorname{sh}(\rho(w))$ for every $w \in \mathscr{D}_{n}$.

Proof. Let $w$ be a Dyck path in $\mathscr{D}_{n}$, we want to construct a Dyck path $\rho(w)$ with $\operatorname{maj}(w)=\operatorname{sh}(\rho(w))$.

We process each down step except the last one, from left to right, as follows (an overline denotes the down step currently being processed).

- If $\bar{D}$ is immediately preceded by a $D$ in the current path, then we do nothing: the updated path is the same as the current one.

- If $\bar{D}$ is preceded by a $U$ in the current path, there are two cases:

1. If $\bar{D}$ is followed by a $U$, interchange this $U$ and $\bar{D}$, i.e., $U \bar{D} U \rightarrow U U \bar{D}$;

2. If $\bar{D}$ is followed by a $D$, let $k$ denote the length of the run of $U$ s preceding $\bar{D}$. If $k=1$, then we do nothing; if $k>1$, interchange $\bar{D}$ and the second $U$ (left to right) in the run, thus $U^{k} \bar{D} D \rightarrow U \bar{D} U^{k-1} D$.

Note that processing $\bar{D}$ does not disturb the later $D$ s. Now we prove that maj $(w)=$ $\operatorname{sh}(\rho(w))$. Assume that $\bar{D}$ is the $i$-th down step. (1) If $\bar{D}$ is immediately preceded by a $D$ and followed by a $U$, then $i \in \mathrm{D}(w)$. Now if we do nothing, then $i \in \mathrm{H}(\rho(w))$; If $\bar{D}$ is immediately preceded by a $D$ and followed by a $D$, then $i \notin \mathrm{D}(w)$. If we do nothing, then $i \notin \mathrm{H}(\rho(w))$. (2) If $\bar{D}$ is immediately preceded by a $U$ and followed by a $U$, then $i \in \mathrm{D}(w)$. In this case, we change $U \bar{D} U$ to $U U \bar{D}$, which implies that $i \in \mathrm{H}(\rho(w))$. (3) If $\bar{D}$ is immediately preceded by a $U$ and followed by a $D$, then $i \notin \mathrm{D}(w)$ but $i$ may belong to $\mathrm{H}(w)$, which depends on the length $k$ of the run of $U$ s preceding $\bar{D}$. If $k=1$, then $i \notin \mathrm{H}(w)$. If we do nothing, then $i \notin \mathrm{H}(\rho(w))$; If $k>1$, then $i \in \mathrm{H}(w)$. In this case we change $U^{k} \bar{D} D$ to $U \bar{D} U^{k-1} D$, which ensures that $i \notin \mathrm{H}(\rho(w))$.

Now we can see that the above process has ensured that maj $(w)=\operatorname{sh}(\rho(w))$.

Finally we define the inverse of $\rho$. For the inverse, we begin from the $(n-1)$-st down step and proceed as follows.

- If $\bar{D}$ is preceded by a $D$ in the current path, we do nothing.

- If $\bar{D}$ is preceded by a $U$ in the current path, let $k$ denote the length of the run of $U$ s preceding the current $\bar{D}$. There are two cases: 
1. If $k>1$, then we interchange $\bar{D}$ and the first $U$ from right to left in the run, thus $U^{k} \bar{D} \rightarrow U^{k-1} \bar{D} U$;

2. If $k=1$ and $\bar{D}$ is followed by a $D$, then we do nothing. If $k=1$ and $\bar{D}$ is followed by a $U$, assume that the length of the run of $U$ s following $\bar{D}$ is $t$, then we interchange $\bar{D}$ and the last $U$ in the run, i.e., $U \bar{D} U^{t} D \rightarrow U^{t+1} \bar{D} D$.

Again note that processing $\bar{D}$ does not disturb the former $D$ s and it is easy to check that the above algorithm is the inverse of $\rho$.

As a corollary of Theorem 2.1, we have

Corollary 2.2 For each $n>0$, we have

$$
\sum_{w \in \mathscr{D}_{n}} t^{v(w)} q^{\operatorname{maj}(w)}=\sum_{w \in \mathscr{D}_{n}} t^{h(w)} q^{\operatorname{sh}(w)},
$$

where $v(w)$ (resp., $h(w)$ ) denotes the number of valleys (resp., skew hooks) of $w$.

Example 2.3 Here we give a Dyck path $w \in \mathscr{D}_{6}$ and its corresponding Dyck path $\rho(w)$ in Figure 2. The process is as follows: $U \bar{D} U^{3} D^{2} U^{2} D^{3} \rightarrow U^{2} D U^{2} \bar{D} D U^{2} D^{3} \rightarrow$ $U^{2} D U D U \bar{D} U^{2} D^{3} \rightarrow U^{2} D U D U U D U \bar{D} D D \rightarrow U^{2} D U D U U D U D \bar{D} D$.

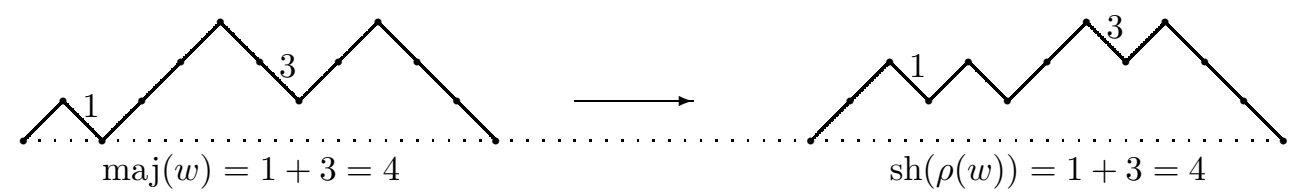

Figure 2: Illustration of Theorem 2.1

\section{Statistics on Non-crossing Partitions}

In this section we present an investigation of the relations between the statistics $l s, r b$ on $N C(n, k+1)$ and the statistic maj on $\mathscr{D}_{n, k}$.

First we recall the well known bijection between Dyck paths and non-crossing partitions $[6,8]$. For a given non-crossing partition $\pi \in N C(n, k)$, arrange the blocks of $\pi$ in increasing order of their maximal elements. Let $m_{i}(1 \leq i \leq k)$ be these $k$ maximal elements and let $h_{i}(1 \leq i \leq k)$ be the corresponding block sizes. We assume that $m_{0}=0$. Then the pairs $\left(m_{i}-m_{i-1}, h_{i}\right)_{i=1}^{k}$ determine a Dyck path of length $2 n$ as follows: for each $i$, we first put $m_{i}-m_{i-1}$ up steps and then we put $h_{i}$ down steps to follow these up steps immediately. For the inverse mapping, we label the up steps of the Dyck path by enumerating them from left to right (so that the $k$-th up step is labeled $k$ ). Next assign to each down step the same label of its matching up step. The numbers assigned on a maximal sequence of continuous down steps form a block of the desired non-crossing partition. We give an example illustrated in Figure 3.

In the rest of this paper, representing a Dyck path as a non-crossing partition means the bijection described above. 


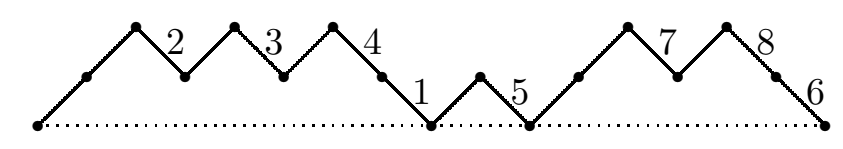

Figure 3: The corresponding non-crossing partition is $14 / 2 / 3 / 5 / 68 / 7$.

\subsection{Bijection between $r b$ and maj}

Now we prove that the statistic $r b$ on non-crossing partitions is equivalent to the statistic maj defined on Dyck paths.

Definition 3.1 A non-crossing partition $\pi \in N C(n)$ is primitive if 1 and $n$ belong to the same block of $\pi$.

Definition 3.2 A primitive Dyck path is one that returns the path to the $x$-axis exactly once.

We can represent non-crossing partitions graphically, plotting $1,2, \ldots, n$ on a horizontal line and joining successive elements of the same block by arcs. For example, we can represent $\pi=18 / 24 / 3 / 57 / 6$ as follows:

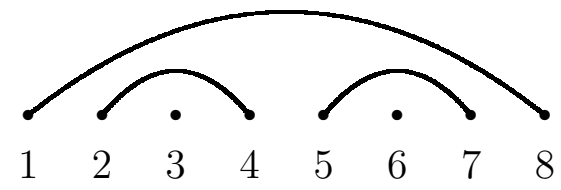

The least (resp., greatest) element of a block is called a left-hand (resp., right-hand) endpoint. If the size of a block is one, then the unique element of this block is called a singleton. Here we note that a singleton can be viewed as a left-hand endpoint and also can be viewed as a right-hand endpoint.

We denote $l_{i}(\pi)$ (resp., $\left.r_{i}(\pi)\right)$ the $i$-th left-hand endpoint (resp., right-hand endpoint) of the partition $\pi$. Let $b_{i}(\pi)$ denote the size of the block containing the element $r_{i}$. Hence in the partition mentioned above, we have $l_{1}(\pi)=1, l_{2}(\pi)=2, l_{3}(\pi)=3, l_{4}(\pi)=5, l_{5}(\pi)=6$; $r_{1}(\pi)=3, r_{2}(\pi)=4, r_{3}(\pi)=6, r_{4}(\pi)=7, r_{5}(\pi)=8$; and $b_{1}(\pi)=1, b_{2}(\pi)=2, b_{3}(\pi)=$ $1, b_{4}(\pi)=2, b_{5}(\pi)=2$.

Lemma 3.3 Assume $\pi \in N C(n, k)$ and $n>1$. The following conditions are equivalent:

(a) $\pi$ is primitive;

(b) $1=l_{1}(\pi)<l_{2}(\pi)<\cdots<l_{k}(\pi)<n, 1<r_{1}(\pi)<r_{2}(\pi)<\cdots<r_{k}(\pi)=n$, where $1 \leq k \leq n-1$, and $r_{i}(\pi) \geq l_{i+1}(\pi)$ for $1 \leq i \leq k-1$;

(c) $1 \leq k \leq n-1$ and $b_{1}(\pi)+\cdots+b_{i}(\pi)<r_{i}(\pi)$ for each $i<k$. 
Proof. $(a) \Rightarrow(b)$ and $(c)$ : if $\pi$ has $n$ blocks, then every element of $[n]$ consists a block by itself, so 1 and $n$ can not lie in the same block. Hence the number of blocks must be less than $n$, that is, $k<n$. For any partition $\pi$, we always have

$$
1=l_{1}(\pi)<l_{2}(\pi)<\cdots<l_{k}(\pi) \text { and } r_{1}(\pi)<r_{2}(\pi)<\cdots<r_{k}(\pi)=n \text {. }
$$

If $l_{k}(\pi)=n$, then $n$ is a singleton. If $r_{1}(\pi)=1$, then 1 is a singleton. Hence if $\pi$ is primitive, we must have

$$
1=l_{1}(\pi)<l_{2}(\pi)<\cdots<l_{k}(\pi)<n \text { and } 1<r_{1}(\pi)<r_{2}(\pi)<\cdots<r_{k}(\pi)=n .
$$

We now prove that $r_{i}(\pi) \geq l_{i+1}(\pi)$ and $b_{1}(\pi)+\cdots+b_{i}(\pi)<r_{i}(\pi)$ for every $i<k$. Assume that $t$ is the least $i$ such that $r_{i}(\pi)<l_{i+1}(\pi)$, then there are $t$ left-hand endpoints and $t$ right-hand endpoints in the interval between 1 and $r_{t}$. Since $\pi$ is a partition, for every right-hand endpoint, there exists a left-hand endpoint to be matched with it. The condition

$$
r_{1}(\pi) \geq l_{2}(\pi), r_{2}(\pi) \geq l_{3}(\pi), \ldots, r_{t-1}(\pi) \geq l_{t}(\pi) \text { and } r_{t}(\pi)<l_{t+1}(\pi)
$$

and $t$ is the least $i$ such that $r_{i}(\pi)<l_{i+1}(\pi)$ ensures that 1 is in the same block with $r_{t}(\pi)$, which contradicts with the fact that $\pi$ is primitive.

It is easy to see that $b_{1}(\pi)+\cdots+b_{i}(\pi) \leq r_{i}(\pi)$ for every $i<k$. We claim that for a primitive partition, the equality can not hold. If there exists some $i$ such that $b_{1}(\pi)+$ $\cdots+b_{i}(\pi)=r_{i}(\pi)$, assume that the least such $i$ is $t$, then the elements $\left\{1,2, \ldots, r_{t}(\pi)\right\}$ have formed a primitive partition of $\left[r_{t}(\pi)\right]$.

$(b) \Rightarrow(a): r_{1}(\pi)>1$ (resp., $l_{k}(\pi)<n$ ) shows that 1 (resp., $n$ ) is not a singleton. We assume that the greatest element of the block containing 1 is $r_{s}(\pi)$. If $\pi$ is not primitive, then $r_{s}(\pi)<n=r_{k}(\pi)$ and $s<k$. Since $\pi$ is non-crossing, then $r_{s}(\pi)+1$ must be a left-hand point and $r_{s}(\pi)+1=l_{s+1}(\pi)$, which yields that $r_{s}(\pi)<l_{s+1}(\pi)$.

$(c) \Rightarrow(a)$ : We also assume that the greatest element of the block containing 1 is $r_{s}(\pi)$. If $\pi$ is not primitive, then $r_{s}(\pi)<n=r_{k}(\pi)$ and $s<k$; and $b_{1}(\pi)+\cdots+b_{s}(\pi)=r_{s}(\pi)$, which yields a contradiction.

Remark. (I) If we have a date set $\left\{l_{i}, r_{i}\right\}$ for $1 \leq i \leq k$ and $k<n$ such that

$$
1=l_{1}<l_{2}<\cdots<l_{k}<n, 1<r_{1}<r_{2}<\cdots<r_{k}=n \text { and } r_{i} \geq l_{i+1} \text { for } 1 \leq i \leq k-1 \text {, }
$$

we can uniquely construct a partition $\pi$ such that $\pi \in N C(n, k)$ and $l_{i}(\pi)=l_{i}$ and $r_{i}(\pi)=r_{i}$ for $1 \leq i \leq k$ as follows.

We first parenthesize the sequence $1,2, \ldots, n$ by placing a left (resp., right) parenthesis before every $l_{i}$ (resp., after every $r_{i}$ ) for $1 \leq i \leq k$. Then create a block of $\pi$ for each of the consecutive strings inside "lowest level" parenthesis pairs (i.e., parentheses which pair each other and enclose no others). Now remove these lowest level parenthesis pairs and all the numbers they enclose, and continue with the remaining parenthesization. Clearly the partition $\pi$ yielded above is non-crossing and has $k$ blocks. Since $r_{i} \geq l_{i+1}$ for $1 \leq i \leq k-1$, there are $k$ left parentheses before the $(k-1)$-st right parenthesis, hence 
the left parenthesis before 1 must be paired with the right parenthesis after $n$, i.e., 1 and $n$ belong to the same block, which means that the partition $\pi$ is primitive.

(II) If we have a date set $\left\{r_{i}, b_{i}\right\}$ for $1 \leq i \leq k$ and $k<n$ such that

$$
1<r_{1}<r_{2}<\cdots<r_{k}=n \text { and } b_{1}+\cdots+b_{i}<r_{i} \text { for } 1 \leq i \leq k-1 \text {, }
$$

we can also uniquely construct a partition $\pi$ such that $\pi \in N C(n, k), r_{i}(\pi)=r_{i}$ and $b_{i}(\pi)=b_{i}$ for $1 \leq i \leq k$ as follows.

We first plot $1,2, \ldots, n$ on the horizontal line, then start from the number $r_{1}$ and count successively $b_{1}$ numbers from right to left, so we get a block of $\pi$ with $r_{1}$ being its greatest element and size of $b_{1}$. We delete these selected $b_{1}$ numbers and start from the number $r_{2}$ and choose $b_{2}$ rightmost unselected numbers before $r_{2}$ to form another block of $\pi$. By iterating this process, we get $k$ blocks of $\pi$ and clearly $\pi$ is non-crossing. Since $b_{1}+\cdots+b_{i}<r_{i}$ for $1 \leq i \leq k-1$, after we create the $(k-1)$-st block of $\pi, 1$ must have not been selected, hence 1 and $n$ will be put together to form the $k$-th block, which means that $\pi$ is primitive.

Theorem 3.4 There is a bijection $\gamma: N C(n, k+1) \rightarrow \mathscr{D}_{n, k}$ such that if $\pi \in N C(n, k+1)$, then $\operatorname{rb}(\pi)=\operatorname{maj}(\gamma(\pi))$.

Proof. If a non-crossing partition $\pi$ has only one block of size $n$, i.e., $\pi=\{1,2, \ldots, n\}$, then we define $\gamma(\pi)=U_{1} U_{2} \cdots U_{n} D_{1} D_{2} \cdots D_{n}$. This case is trivial.

Now we consider the nontrivial cases.

We first define our bijection $\gamma$ between the set of primitive non-crossing partitions and the set of primitive Dyck paths.

Assume that $\pi \in N C(n, k+1)$. We let $\bar{r}_{1}=r_{1}(\pi), \bar{r}_{2}=r_{2}(\pi), \ldots, \bar{r}_{k}=r_{k}(\pi)$ and $\bar{b}_{1}=l_{2}(\pi)-1, \bar{b}_{2}=l_{3}(\pi)-l_{2}(\pi), \bar{b}_{3}=l_{4}(\pi)-l_{3}(\pi), \ldots, \bar{b}_{k}=l_{k+1}(\pi)-l_{k}(\pi)$. Since $\pi$ is primitive, from Lemma 3.3 we know that $r_{i}(\pi) \geq l_{i+1}(\pi)$ for $1 \leq i \leq k$ which implies that

$$
\bar{b}_{1}+\bar{b}_{2}+\cdots+\bar{b}_{i}=l_{i+1}(\pi)-1<l_{i+1}(\pi) \leq r_{i}(\pi)=\bar{r}_{i} \text { for } 1 \leq i \leq k .
$$

From the case (II) in the remark before Theorem 3.4, we know that there exists a unique non-crossing partition $\bar{\pi}$ satisfying that

$$
r_{1}(\bar{\pi})=\bar{r}_{1}, r_{2}(\bar{\pi})=\bar{r}_{2}, \ldots, r_{k+1}(\bar{\pi})=\bar{r}_{k+1} \text { and } b_{1}(\bar{\pi})=\bar{b}_{1}, b_{2}(\bar{\pi})=\bar{b}_{2}, \ldots, b_{k+1}(\bar{\pi})=\bar{b}_{k+1} \text {. }
$$

Finally we represent $\bar{\pi}$ as a Dyck path, which is the desired Dyck path $\gamma(\pi)$.

Given a primitive Dyck path $w \in \mathscr{D}_{n, k}$, we can associate it with a non-crossing partition $\bar{\pi} \in N C(n, k+1)$. From the bijection between Dyck paths and non-crossing partitions constructed in [6], we know that $\bar{\pi}$ is primitive. We let

$$
r_{1}=r_{1}(\bar{\pi}), r_{2}=r_{2}(\bar{\pi}), \ldots, r_{k}(\pi)=r_{k}(\bar{\pi})
$$

and

$$
l_{2}=b_{1}(\bar{\pi})+1, l_{3}=b_{2}(\bar{\pi})+l_{2}(\pi), \ldots, l_{k+1}=b_{k}(\bar{\pi})+l_{k}(\pi) .
$$


From the primitiveness of $\bar{\pi}$ we can derive that

$$
b_{1}(\bar{\pi})+b_{2}(\bar{\pi})+\cdots+b_{i}(\bar{\pi})<r_{i}(\bar{\pi}) \text { for } 1 \leq i \leq k,
$$

which shows that

$$
l_{i+1}-1=\left(l_{2}-1\right)+\left(l_{3}-l_{2}\right)+\cdots+\left(l_{i+1}-l_{i}\right)<r_{i}(\bar{\pi})=r_{i} \text { for } 1 \leq i \leq k,
$$

that is, $l_{i+1} \leq r_{i}$ for each $1 \leq i \leq k$. From the case (I) in the remark before Theorem 3.4, we know that there exists a unique non-crossing partition $\pi$ such that

$$
l_{1}(\pi)=l_{1}, l_{2}(\pi)=l_{2}, \ldots, l_{k+1}(\pi)=l_{k+1}
$$

and

$$
r_{1}(\pi)=r_{1}, r_{2}(\pi)=r_{2}, \ldots, r_{k+1}(\pi)=r_{k+1}
$$

Since

$$
\begin{aligned}
r b(\pi)= & \sum_{i=1}^{k+1}\left(m_{i}(\pi)-1\right) \\
= & \sum_{i=2}^{k+1}\left(l_{i}(\pi)-1\right) \\
= & b_{1}(\bar{\pi})+\left(b_{1}(\bar{\pi})+b_{2}(\bar{\pi})\right)+\left(b_{1}(\bar{\pi})+b_{2}(\bar{\pi})+b_{3}(\bar{\pi})\right)+\cdots \\
& +\left(b_{1}(\bar{\pi})+b_{2}(\bar{\pi})+b_{3}(\bar{\pi})+\cdots+b_{k}(\bar{\pi})\right) \\
= & \operatorname{maj}(\gamma(\pi)),
\end{aligned}
$$

so $\operatorname{rb}(\pi)=\operatorname{maj}(\gamma(\pi))$.

If the non-crossing partition is not primitive, then we can represent it as the union of several primitive non-crossing partitions and each such primitive non-crossing partition corresponds to a primitive Dyck path. By concatenating these Dyck paths in the order of those primitive non-crossing partitions, we get a Dyck path. It is straightforward to check that this is the desired Dyck path.

Example 3.5 Let $\pi=18 / 24 / 3 / 57 / 6$, we have known that $w(\pi)=12324541$ and $r b(\pi)=12$. We construct a non-crossing partition $\bar{\pi}$ and its corresponding Dyck path $\gamma(\pi)$ satisfying maj $(\gamma(\pi))=12$ in Figure 4.

\subsection{Bijection between $l s$ and maj}

For the statistic $l s$, the bijection between $N C(n, k+1)$ and $\mathscr{D}_{n, k}$ is relatively simple and elegant.

Theorem 3.6 There is a bijection $\eta: N C(n, k+1) \rightarrow \mathscr{D}_{n, k}$ such that if $\pi \in N C(n, k+1)$, then there is a Dyck path $\eta(\pi) \in \mathscr{D}_{n, k}$ satisfying $l s(\pi)=\operatorname{maj}(\eta(\pi))$. 

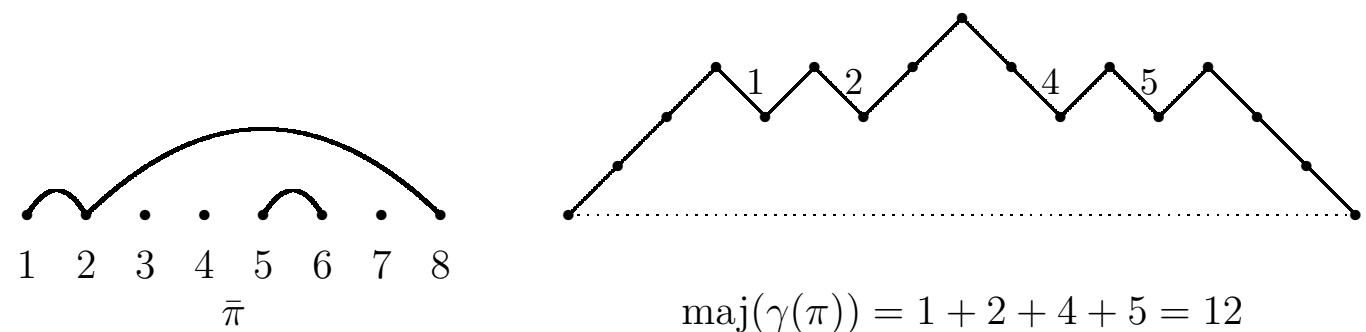

$$
\operatorname{maj}(\gamma(\pi))=1+2+4+5=12
$$

Figure 4: The bijection $\gamma(\pi)$.

Proof. For $\pi \in N C(n, k+1)$, we change $i$ to $n+1-i$, then we get a partition $\bar{\pi}$. It is easy to see that $\bar{\pi}$ is also a non-crossing partition in $N C(n, k+1)$. We associate $\bar{\pi}$ with the corresponding Dyck path, then we get the desired Dyck path $\eta(\pi)$. It is obviously that $\eta$ is invertible. So we only have to show that $l s(\pi)=\operatorname{maj}(\eta(\pi))$. We can derive that

$$
\begin{aligned}
\operatorname{maj}(\eta(\pi)) & =\sum_{i \in \mathrm{D}(\eta(\pi))} i=\sum_{i=1}^{k}(k+1-i) b_{i}(\bar{\pi}) \\
& =\sum_{i=1}^{k}(k+1-i)\left|B_{k+2-i}(\pi)\right|=\sum_{i=1}^{k}(k+2-i-1)\left|B_{k+2-i}(\pi)\right| \\
& =\sum_{i=2}^{k+1}(i-1)\left|B_{i}(\pi)\right|=\sum_{i=1}^{k+1}(i-1)\left|B_{i}(\pi)\right|=l s(\pi),
\end{aligned}
$$

where $b_{i}(\pi)$ denotes the size of the block containing the $i$-th right-hand endpoint of $\pi$.

\section{4 q-Narayana Numbers and $q$-Catalan Numbers}

In [4], Fürlinger and Hofbauer gave the most obvious q-analogue of the Narayana numbers and the Catalan numbers based on Dyck paths. Also there are many other research articles devoted to the $q$-analogues of the Narayana numbers and the Catalan numbers. For example, see [3] for $q$-analogue of the Narayana numbers with other statistics on Dyck paths, and Andrews [1] produced some simple $q$-analogues of the Catalan numbers related to the theory of partitions.

Using the statistic Maj on Dyck paths, Fürlinger and Hofbauer [4] gave the $q$-analogue of the Narayana numbers and the Catalan numbers:

$$
\begin{aligned}
\sum_{w \in \mathscr{D}_{n, k}} q^{\operatorname{Maj}(w)} & =\frac{1}{[n]}\left[\begin{array}{c}
n \\
k+1
\end{array}\right]\left[\begin{array}{l}
n \\
k
\end{array}\right] q^{k^{2}+k}, \\
\sum_{w \in \mathscr{D}_{n}} q^{\operatorname{Maj}(w)} & =\frac{1}{[n+1]}\left[\begin{array}{c}
2 n \\
n
\end{array}\right] .
\end{aligned}
$$


In this section, we will give the same $q$-analogue of the Narayana numbers and the Catalan numbers using the statistics $l s, r b$ on non-crossing partitions.

For the convenience of reader, we give a short description of two statistics $\alpha$ and $\beta$ defined in [4, Section 5]. If $i \in \mathrm{D}(w)$, let $\alpha(i)$ denote the number of up steps before $D_{i}$ and $\beta(i)$ denote the number of down steps before $D_{i}$ and including $D_{i}$. For $w \in \mathscr{D}_{n}$, Fürlinger and Hofbauer [4] defined

$$
\alpha(w):=\sum_{i \in \mathrm{D}(w)} \alpha(i) \quad \text { and } \quad \beta(w):=\sum_{i \in \mathrm{D}(w)} \beta(i) .
$$

In fact, our maj defined on Dyck paths is equivalent to $\beta$. Under the bijection $\eta$, the statistic $l s$ defined on non-crossing partitions is also equivalent to $\beta$. Our goal of this section is to find another statistic, which is equivalent to $\alpha$.

Lemma 4.1 Given $\pi \in N C(n)$ and let bk( $\pi)$ denote the number of blocks of $\pi$, then $(b k(\pi)-1) n-r b(\pi)$ is equivalent to $\alpha$.

Proof. Given a non-crossing partition $\pi \in N C(n, k)$, i.e., $\pi$ has exactly $k$ blocks, represent it as $\pi=B_{1} / B_{2} / \cdots / B_{k}$. Under the bijection $\eta$, we get another non-crossing partition $\bar{\pi}=\bar{B}_{1} / \bar{B}_{2} / \cdots / \bar{B}_{k}$. If we associate $\bar{\pi}$ with a Dyck path $w$, clearly there are exactly $k$ peaks and $k-1$ valleys on $w$. From the bijection between non-crossing partition $\bar{\pi}$ and Dyck path $w$, we know that

$$
\sum_{i=1}^{k} M_{i}(\bar{\pi})-n=\alpha(w)
$$

where $M_{i}(\bar{\pi})=\max \left\{a: a \in \bar{B}_{i}\right\}$. Furthermore, under the bijection $\eta$, we get

$$
\sum_{i=1}^{k} M_{i}(\bar{\pi})=\sum_{i=1}^{k}\left(n+1-m_{i}(\pi)\right)
$$

where $m_{i}(\pi)=\min \left\{a: a \in B_{i}\right\}$. And now we can have

$$
\begin{aligned}
\alpha(w) & =\sum_{i=1}^{k} M_{i}(\bar{\pi})-n=\sum_{i=1}^{k}\left(n+1-m_{i}(\pi)\right)-n \\
& =\sum_{i=2}^{k}\left(n+1-m_{i}(\pi)\right)=\sum_{i=2}^{k}\left\{n-\left(m_{i}(\pi)-1\right)\right\} \\
& =(k-1) n-\sum_{i=2}^{k}\left(m_{i}(\pi)-1\right)=(k-1) n-\sum_{i=1}^{k}\left(m_{i}(\pi)-1\right) \\
& =(k-1) n-r b(\pi)=(b k(\pi)-1) n-r b(\pi) .
\end{aligned}
$$

From the above analysis we can also derive a $q$-Narayana distribution on non-crossing partitions. 


\section{Theorem 4.2}

$$
\sum_{\pi \in N C(n, k)} q^{(k-1) n+l s(\pi)-r b(\pi)}=\frac{1}{[n]}\left[\begin{array}{l}
n \\
k
\end{array}\right]\left[\begin{array}{c}
n \\
k-1
\end{array}\right] q^{k^{2}-k} .
$$

Proof. As we know the bijection $\eta$ between $\pi \in N C(n, k)$ and $w \in \mathscr{D}_{n, k-1}$ transforms the statistic $l s(\pi)$ to statistic $\beta(w)$ and $(k-1) n-r b(\pi)$ to $\alpha(w)$, thus

$$
(k-1) n+l s(\pi)-r b(\pi)=\alpha(w)+\beta(w)=\operatorname{Maj}(w) .
$$

Invoking identity (4.1) in [4, Section 4], we obtain the following identity:

$$
\sum_{\pi \in N C(n, k)} q^{(k-1) n+l s(\pi)-r b(\pi)}=\frac{1}{[n]}\left[\begin{array}{l}
n \\
k
\end{array}\right]\left[\begin{array}{c}
n \\
k-1
\end{array}\right] q^{k^{2}-k} .
$$

This completes the proof.

Summing over $k$ from 1 to $n$ yields the $q$-analogue of the Catalan numbers.

\section{Corollary 4.3}

$$
\sum_{\pi \in N C(n)} q^{(b k(\pi)-1) n+l s(\pi)-r b(\pi)}=\frac{1}{[n+1]}\left[\begin{array}{c}
2 n \\
n
\end{array}\right] .
$$

Acknowledgments. The authors are grateful to the Editor Catherine Yan and the anonymous referee for their thoughtful and insightful comments that have vastly improved this paper.

\section{References}

[1] G.E. Andrews, Catalan numbers, $q$-Catalan numbers and hypergeometric series, $J$. Combin. Theory Ser. A, 44 (1987), 267-273.

[2] S. Benchekroun and P. Moszkowski, A bijective proof of an enumerative property of legal bracketings, Discrete Math., 176 (1997), 273-277.

[3] P. Branden, $q$-Narayana number and the flag $h$-vector of $J(2 \times n)$, Discrete Math., 281 (2004), 67-81.

[4] J. Fürlinger and J. Hofbauer, q-Catalan numbers, J. Combin. Theory Ser. A, 40 (1985), 248-264.

[5] S. Milne, Restricted growth functions, ranks row matchings of partition lattices, and q-Stirling numbers, Adv. in Math., 43 (1982), 173-196.

[6] H. Prodinger, A correspondence between ordered trees and noncrossing partitions, Discrete Math., 46 (1983), 205-206.

[7] R. Simion, Combinatorial statistics on non-crossing partitions, J. Combin. Theory Ser. A, 66 (1994), 270-301.

[8] R.P. Stanley, "Enumerative Combinatorics", Vol 2, Cambridge University Press, Cambridge, New York, 1999. 